\title{
Interaction of the components in the system $\mathrm{Hf}-\mathrm{Re}-\mathrm{Si}$
}

\author{
Liana ZINKO ${ }^{1}$, Oksana MATSELKO $^{1 *}$, Vasyl KORDAN ${ }^{1}$, Galyna NYCHYPORUK $^{1}$, Roman GLADYSHEVSKII $^{1}$ \\ ${ }^{1}$ Department of Inorganic Chemistry, Ivan Franko National University of Lviv, \\ Kyryla i Mefodiya St. 6, 79005 Lviv, Ukraine \\ * Corresponding author. Tel.: +380-32-2394506; e-mail: oksana.matselko@lnu.edu.ua
}

Received October 25, 2019; accepted December 18, 2019; available on-line April 1, 2020 https://doi.org/10.30970/cma12.0398

The interaction of the components in the ternary system $\mathrm{Hf}-\mathrm{Re}-\mathrm{Si}$ at $1000^{\circ} \mathrm{C}$ has been investigated by means of X-ray powder diffraction and scanning electron microscopy with energy-dispersive X-ray spectroscopy. The system is characterized by the formation of solid solutions based on the binary phases. In addition to HfReSi and HfReSi $i_{2}$, two more ternary phases, with approximate compositions $\mathrm{Hf}_{23(2)} \operatorname{Re}_{4(1)} \mathrm{Si}_{73(1)}(\mathrm{T1})$ and $\mathrm{Hf}_{71(2)} \operatorname{Re}_{18(3)} \mathrm{Si}_{11(1)}(\mathrm{T} 2)$, were observed. According to the EDXS analysis, the highest solubility of the third component was found for $\mathrm{HfRe}_{2}$ (up to 36.4 at.\% $\mathrm{Si}$ ) and $\mathrm{Re}_{4} \mathrm{Si}_{7}$ (up to 23.8 at.\% $\mathrm{Hf}$ ). The ternary phase HfReSi exhibits a significant homogeneity range with an approximately constant $\mathrm{Hf}$ content at $1000^{\circ} \mathrm{C}$ $\left(\mathrm{Hf}_{29.1(3)-25.0(4)} \operatorname{Re}_{30.3(5)-45(2)} \mathrm{Si}_{40.6(8)-30(2)}\right)$.

Hafnium / Rhenium / Silicon / X-ray powder diffraction / Scanning electron microscopy

\section{Introduction}

Low thermal expansion is an important characteristic of construction materials. The peculiar properties of some of the Hf-Si and Re-Si compounds [1] make the ternary $\mathrm{Hf}-\mathrm{Re}-\mathrm{Si}$ system attractive for a detailed investigation of the interaction of the components at elevated temperatures, and the consecutive construction of isothermal sections of the phase diagram.

The systems $\{\mathrm{Ti}, \mathrm{Zr}, \mathrm{Hf}\}-\{\mathrm{Mn}, \mathrm{Re}\}-\{\mathrm{Si}, \mathrm{Ge}, \mathrm{Sn}, \mathrm{Pb}\}$, or $T^{\mathrm{IV}}-T^{\mathrm{VII}}-M^{\mathrm{IV}}$, have not yet been thoroughly investigated. Of the 24 ternary systems, isothermal sections of the phase diagrams (mainly partial, experimental) have been constructed only for the $\mathrm{Ti}-\mathrm{Mn}-\{\mathrm{Si}, \mathrm{Ge}, \mathrm{Sn}\}$ systems [2]. 32 ternary compounds, representing 13 structure types, 9 of them fully ordered, have been reported in the $T^{\mathrm{IV}}-T^{\mathrm{VII}}-M^{\mathrm{IV}}$ systems ([3], Table 1). It should be noted that almost all of the known compounds contain $\mathrm{Mn}$ and the largest number of representatives, eight, has the structure type $\mathrm{ZrCrSi}_{2}$.

As for the Hf-Re-Si system in particular, two ternary compounds - HfReSi (structure type ZrNiAl, space group $P-62 m$, Pearson symbol $h P 9$ ) [4] and $\mathrm{HfReSi}_{2}$ ( $\mathrm{ZrCrSi}_{2}$, Pbam, oP48) [5] - have been reported. The boundary systems, Hf-Re, Hf-Si, and $\mathrm{Re}-\mathrm{Si}$, are well known and their phase diagrams have been constructed [6]. Crystallographic parameters of the binary and ternary compounds reported prior to this investigation are summarized in Table 2.

In this work, we present the results of a preliminary investigation of the interaction of the components in the system $\mathrm{Hf}-\mathrm{Re}-\mathrm{Si}$ at $1000^{\circ} \mathrm{C}$.

\section{Experimental}

Alloys were synthesized from high-purity metals (Hf $\geq 99.9$ mass\%, Re $\geq 99.9$ mass\% (pressed pellets), $\mathrm{Si} \geq 99.999$ mass\%) by arc melting with a tungsten electrode, a water-cooled copper hearth and a Ti getter under argon atmosphere. After the synthesis, the ingots were sealed in quartz ampoules under vacuum, annealed at $1000^{\circ} \mathrm{C}$ for 1 week and quenched into cold water. Phase analysis and structure refinements were carried out using X-ray powder diffraction (XRPD) data collected on diffractometers DRON-2.0M (Fe $K \alpha$ radiation) and STOE STADI $\mathrm{P}\left(\mathrm{Cu} K \alpha_{1}\right.$ radiation). The profile and structural parameters were refined by the Rietveld method, using the WinCSD program package [7]. The overall compositions of the samples and of the individual phases, in particular the solubilities of the third component in the binary phases, were investigated by means of energydispersive X-ray spectroscopy (EDXS; scanning electron microscope Tescan Vega 3 LMU equipped with an $\mathrm{X}-\mathrm{Max}^{\mathrm{N}} 20$ silicon drift detector). 
Table 1 Structure types of known compounds in $T^{\mathrm{IV}}-T^{\mathrm{VII}}-M^{\mathrm{IV}}$ systems.

\begin{tabular}{|c|c|c|c|c|c|c|c|c|c|c|c|c|c|c|}
\hline \multicolumn{2}{|c|}{ System $\backslash$ Structure type } & 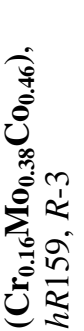 & 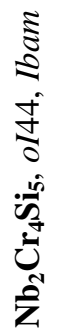 & 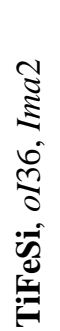 & 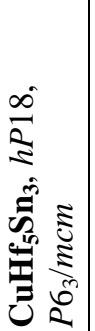 & 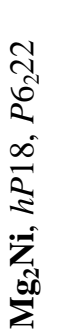 & 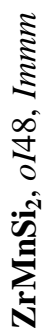 & 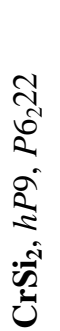 & 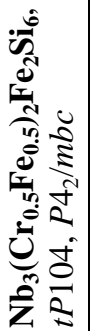 & 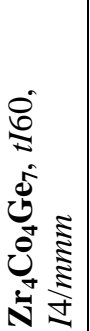 & 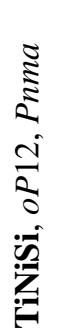 & 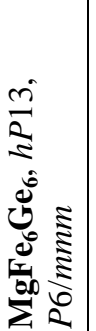 & 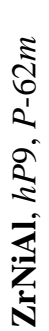 & 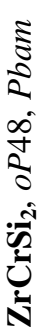 \\
\hline \multirow{4}{*}{$\mathrm{Ti}-\mathrm{Mn}-$} & $\mathrm{Si}$ & & & & & & & & & & & & & \\
\hline & $\mathrm{Ge}$ & & & & & & & & & & & & & \\
\hline & $\mathrm{Sn}$ & & & & & & & & & & & & & \\
\hline & $\mathrm{Pb}$ & & & & & & & & & & & & & \\
\hline \multirow{4}{*}{$\mathrm{Zr}-\mathrm{Mn}-$} & $\mathrm{Si}$ & & & & & & & & & & & & & \\
\hline & $\mathrm{Ge}$ & & & & & & & & & & & & & \\
\hline & $\mathrm{Sn}$ & & & & & & & & & & & & & \\
\hline & $\mathrm{Pb}$ & & & & & & & & & & & & & \\
\hline \multirow{4}{*}{$\mathrm{Hf}-\mathrm{Mn}-$} & $\mathrm{Si}$ & & & & & & & & & & & & & \\
\hline & $\mathrm{Ge}$ & & & & & & & & & & & & & \\
\hline & $\mathrm{Sn}$ & & & & & & & & & & & & & \\
\hline & $\mathrm{Pb}$ & & & & & & & & & & & & & \\
\hline \multirow{4}{*}{ Ti-Re- } & $\mathrm{Si}$ & & & & & & & & & & & & & \\
\hline & $\mathrm{Ge}$ & & & & & & & & & & & & & \\
\hline & $\mathrm{Sn}$ & & & & & & & & & & & & & \\
\hline & $\mathrm{Pb}$ & & & & & & & & & & & & & \\
\hline \multirow{4}{*}{$\mathrm{Zr}-\mathrm{Re}-$} & $\mathrm{Si}$ & & & & & & & & & & & & & \\
\hline & $\mathrm{Ge}$ & & & & & & & & & & & & & \\
\hline & $\mathrm{Sn}$ & & & & & & & & & & & & & \\
\hline & $\mathrm{Pb}$ & & & & & & & & & & & & & \\
\hline \multirow{4}{*}{ Hf-Re- } & $\mathrm{Si}$ & & & & & & & & & & & & & \\
\hline & $\mathrm{Ge}$ & & & & & & & & & & & & & \\
\hline & Sn & & & & & & & & & & & & & \\
\hline & $\mathrm{Pb}$ & & & & & & & & & & & & & \\
\hline \multicolumn{2}{|c|}{ Number of representatives } & 1 & 1 & 1 & 1 & 1 & 1 & 2 & 2 & 2 & 3 & 4 & 5 & 8 \\
\hline
\end{tabular}

\section{Results and discussion}

Twelve ternary alloys were prepared in the system Hf-Re-Si. The results of the analysis by XRPD and EDXS are summarized in Table 3; backscatteredelectron (BSE) images of selected samples, together with the compositions of the identified phases, are shown on Fig. 1.

At $1000^{\circ} \mathrm{C}$, the existence of the binary phases $\mathrm{Hf}_{21} \mathrm{Re}_{25}, \mathrm{HfRe}_{2}, \mathrm{Hf}_{5} \mathrm{Re}_{24}, \mathrm{Hf}_{2} \mathrm{Si}, \mathrm{HfSi}_{2}$, and $\mathrm{Re}_{4} \mathrm{Si}_{7}$, mainly in the form of ternary solid solutions, was confirmed. These findings are in agreement with the assessed binary phase diagrams [20-22]. In addition, we observed a $\mathrm{Hf}_{5} \mathrm{Si}_{3}$-based solid solution. $\mathrm{Hf}_{5} \mathrm{Si}_{3}$ exists between 1718 and $2510^{\circ} \mathrm{C}$ and forms via the peritectic reaction $\mathrm{L}+\mathrm{Hf}_{3} \mathrm{Si}_{2} \leftrightarrow \mathrm{Hf}_{5} \mathrm{Si}_{3}$ at $2510^{\circ} \mathrm{C}$ [23]. At first, this phase was considered to be stabilized by $\mathrm{C}, \mathrm{N}$, or $\mathrm{O}$ [24-26], but later it was found to be stable as a binary phase [27]. We also observed $\mathrm{ReSi}$ (exists between $1650-1820^{\circ} \mathrm{C}$; $\mathrm{L}+\operatorname{ReSi}_{1.75} \leftrightarrow \operatorname{ReSi}$ at $1820^{\circ} \mathrm{C}$ ), while $\mathrm{Hf}_{3} \mathrm{Si}_{2}$
( $\mathrm{L} \leftrightarrow \mathrm{Hf}_{3} \mathrm{Si}_{2}$ at $\left.2480^{\circ} \mathrm{C}\right), \mathrm{Hf}_{5} \mathrm{Si}_{4}\left(\mathrm{~L}+\mathrm{Hf}_{3} \mathrm{Si}_{2} \leftrightarrow \mathrm{Hf}_{5} \mathrm{Si}_{4}\right.$ at $\left.2320^{\circ} \mathrm{C}\right)$, and $\operatorname{Re}_{2} \mathrm{Si}\left(\mathrm{L} \leftrightarrow \operatorname{Re}_{2} \mathrm{Si}\right.$ at $1810^{\circ} \mathrm{C}$ ) were not detected.

The existence of the reported ternary phases HfReSi and $\mathrm{HfReSi}_{2}$ at $1000^{\circ} \mathrm{C}$ was confirmed as well. In addition, two new ternary phases with approximate compositions $\mathrm{Hf}_{23(2)} \mathrm{Re}_{4(1)} \mathrm{Si}_{73(1)}$ (T1) and $\mathrm{Hf}_{71(2)} \mathrm{Re}_{18(3)} \mathrm{Si}_{11(1)}(\mathrm{T} 2)$ were detected.

In the region $63.6-66.7$ at.\% $\mathrm{Si}$ of the system $\mathrm{Re}-\mathrm{Si}$ four compounds with close compositions have been reported: triclinic $\mathrm{ReSi}_{1.75}$, monoclinic $\mathrm{Re}_{4} \mathrm{Si}_{7}$, tetragonal $\mathrm{ReSi}_{1.8}$, and orthorhombic $\mathrm{ReSi}_{2}$. For the Rietveld refinements, performed on multiphase samples, we used the monoclinic crystal structure model.

According to the EDXS analysis, the highest solubilities of the third component were found for $\mathrm{HfRe}_{2}$ (up to 36.4 at.\% Si) and $\mathrm{Re}_{4} \mathrm{Si}_{7}$ (up to 23.8 at.\% Hf). The ternary phase HfReSi showed a significant homogeneity range at an almost constant Hf content $\left(\mathrm{Hf}_{29.1(3)-25.0(4)} \mathrm{Re}_{30.3(5)-45(2)} \mathrm{Si}_{40.6(8)-30(2)}\right)$. 
Table 2 Crystallographic parameters of binary and ternary compounds in the systems $\mathrm{Hf}-\mathrm{Re}, \mathrm{Hf}-\mathrm{Si}, \mathrm{Re}-\mathrm{Si}$, Hf-Re-Si. Binary phases stable at $1000^{\circ} \mathrm{C}$ are highlighted.

\begin{tabular}{|c|c|c|c|c|c|c|c|}
\hline \multirow{2}{*}{ Compound } & \multirow{2}{*}{$\begin{array}{c}\text { Structure } \\
\text { type }\end{array}$} & \multirow{2}{*}{$\begin{array}{c}\text { Pearson } \\
\text { symbol }\end{array}$} & \multirow{2}{*}{$\begin{array}{l}\text { Space } \\
\text { group }\end{array}$} & \multicolumn{3}{|c|}{ Unit-cell parameters, $\AA$} & \multirow{2}{*}{ Ref. } \\
\hline & & & & $a$ & $b$ & $c$ & \\
\hline $\mathbf{H f}_{21} \mathbf{R e}_{25}$ & $\mathrm{Zr}_{21} \mathrm{Re}_{25}$ & $h R 276$ & $R-3 c$ & 25.773 & - & 8.760 & [8] \\
\hline $\mathrm{HfRe}_{2}$ & $\mathrm{MgZn}_{2}$ & $h P 12$ & $\mathrm{PG}_{3} / m m c$ & 5.239 & - & 8.584 & [9] \\
\hline $\mathrm{Hf}_{5} \mathbf{R e}_{24}$ & $\mathrm{Ti}_{5} \mathrm{Re}_{24}$ & cI58 & $I-43 m$ & 9.708 & - & - & [10] \\
\hline $\mathrm{Hf}_{2} \mathrm{Si}$ & $\mathrm{CuAl}_{2}$ & $t I 12$ & $\mathrm{I} 4 / \mathrm{mcm}$ & 6.553 & - & 5.186 & [11] \\
\hline $\mathrm{Hf}_{5} \mathrm{Si}_{3}$ & $\mathrm{Mn}_{5} \mathrm{Si}_{3}$ & $h P 16$ & $\mathrm{PG}_{3} / \mathrm{mcm}$ & 7.844 & - & 5.492 & [12] \\
\hline $\mathbf{H f}_{3} \mathbf{S i}_{2}$ & $\mathrm{U}_{3} \mathrm{Si}_{2}$ & $t P 10$ & $P 4 / m b m$ & 6.988 & - & 3.675 & [12] \\
\hline $\mathrm{Hf}_{5} \mathrm{Si}_{4}$ & $\mathrm{Zr}_{5} \mathrm{Si}_{4}$ & $t P 36$ & $P 4_{1} 2_{1} 2$ & 7.039 & - & 12.83 & [12] \\
\hline HfSi & $\mathrm{FeB}$ & $o P 8$ & Pnma & 6.889 & 3.772 & 5.223 & [12] \\
\hline $\mathrm{HfSi}_{2}$ & $\mathrm{ZrSi}_{2}$ & $o S 12$ & $\mathrm{Cmcm}$ & 3.672 & 14.57 & 3.641 & [12] \\
\hline $\operatorname{Re}_{2} \mathbf{S i}$ & $\mathrm{Re}_{2} \mathrm{Si}$ & $m P 24$ & $P 2_{1} / c$ & 6.4444 & $\begin{array}{c}9.6019 \\
\beta=94.214^{\circ}\end{array}$ & 5.3898 & [13] \\
\hline $\mathrm{Re}_{5} \mathrm{Si}_{3}$ & $\mathrm{~W}_{5} \mathrm{Si}_{3}$ & $t I 32$ & $\mathrm{I} 4 / \mathrm{mcm}$ & 9.53 & - & 4.81 & [14] \\
\hline $\mathrm{ReSi}$ & $\mathrm{FeSi}$ & $c P 8$ & $P 2_{1} 3$ & 4.7744 & - & - & [15] \\
\hline $\mathbf{R e}_{4} \mathbf{S i}_{7}$ & $\mathrm{Re}_{4} \mathrm{Si}_{7}$ & $m S 44$ & $\mathrm{Cm}$ & 23.167 & $\beta=\begin{array}{l}3.14 \\
\beta=94.214^{\circ}\end{array}$ & 8.3018 & [16] \\
\hline $\operatorname{ReSi}_{1.75}$ & $\operatorname{ReSi}_{1.75}$ & $a P 6$ & $P 1$ & $\begin{array}{c}3.12 \\
\alpha=90^{\circ}\end{array}$ & $\begin{array}{c}3.138 \\
\beta=90.1^{\circ}\end{array}$ & $\begin{array}{c}7.67 \\
\gamma=90^{\circ}\end{array}$ & [17] \\
\hline $\operatorname{ReSi}_{1.8}$ & $\mathrm{MoSi}_{2}$ & $t I 6$ & $\mathrm{I} / \mathrm{mmm}$ & 3.132 & - & 7.681 & [18] \\
\hline $\mathrm{ReSi}_{2}$ & $\mathrm{ReSi}_{2}$ & $o I 6$ & Immm & 3.128 & 3.144 & 7.677 & [18] \\
\hline HfReSi & $\mathrm{ZrNiAl}$ & $h P 9$ & $P-62 m$ & 6.927 & - & 3.391 & [4] \\
\hline $\mathrm{HfReSi}_{2}$ & $\mathrm{ZrCrSi}_{2}$ & $o P 48$ & Pbam & 9.106 & 10.016 & 8.062 & [5] \\
\hline
\end{tabular}

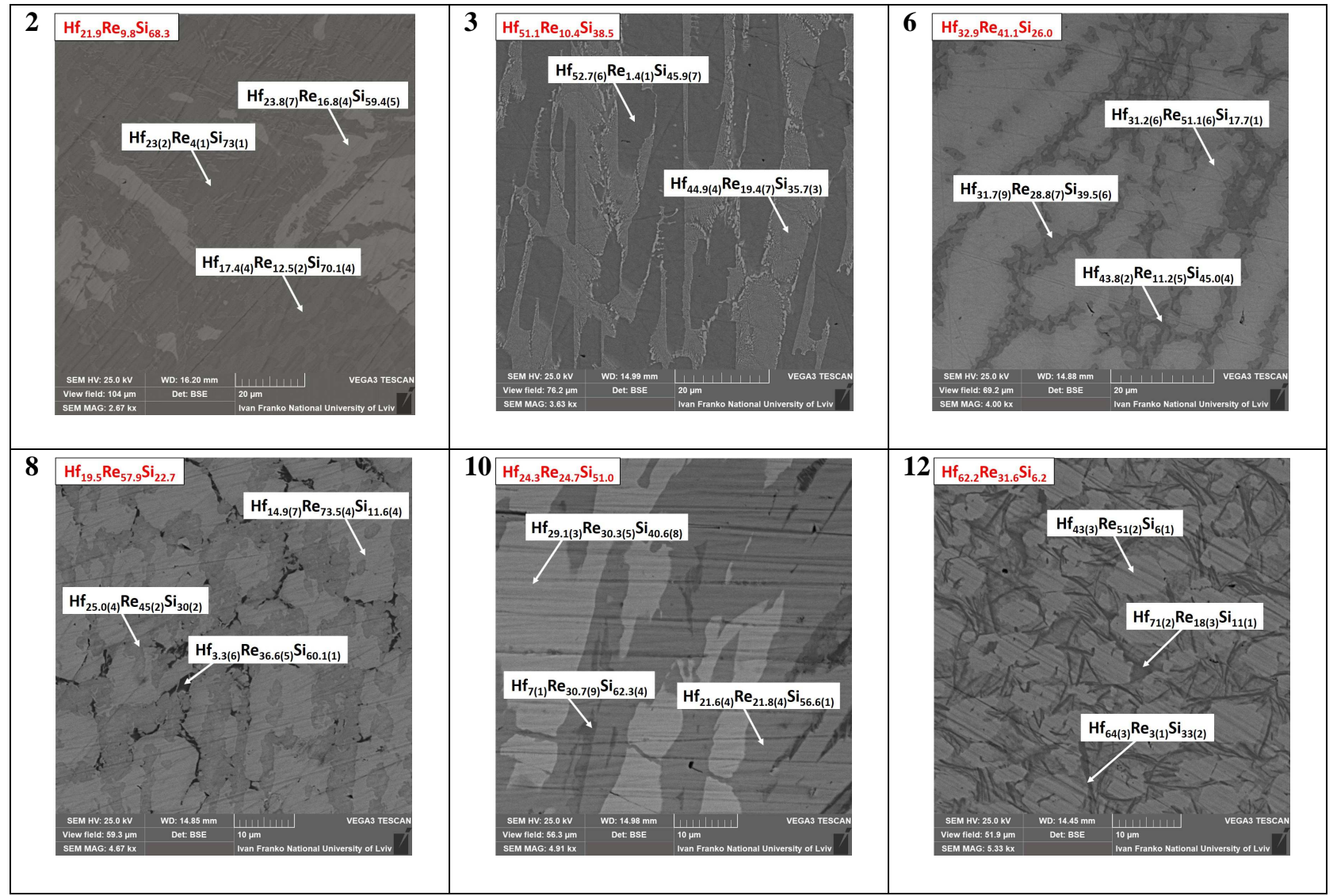

Fig. 1 Microstructures of selected samples (BSE images) and compositions of the observed phases. 
Table 3 Results of the phase analysis of the synthesized samples.

\begin{tabular}{|c|c|c|c|c|c|c|c|}
\hline \multirow{2}{*}{\multicolumn{2}{|c|}{ Sample $^{a}$}} & \multirow{2}{*}{ Phase $^{b}$} & \multirow{2}{*}{$\begin{array}{c}\text { Pearson } \\
\text { symbol }\end{array}$} & \multirow{2}{*}{$\begin{array}{l}\text { Space } \\
\text { group }\end{array}$} & \multicolumn{3}{|c|}{ Unit-cell parameters, $\AA$} \\
\hline & & & & & $a$ & $b$ & $c$ \\
\hline 1 & $\mathrm{Hf}_{15} \mathrm{Re}_{15} \mathrm{Si}_{70}$ & $\begin{array}{l}\mathrm{Re}_{4} \mathrm{Si}_{7} \\
\mathrm{HfSi}_{2} \\
\mathrm{Si}\end{array}$ & $\begin{array}{c}m S 44 \\
o S 12 \\
c F 8\end{array}$ & $\begin{array}{c}\mathrm{Cm} \\
\mathrm{Cmcm} \\
\mathrm{Fd}-3 m\end{array}$ & $\begin{array}{c}23.1718(5) \\
3.67478(9) \\
5.4237(5)\end{array}$ & $\begin{array}{c}3.13614(6) \\
\beta=92.735(3)^{\circ} \\
14.5380(4) \\
-\end{array}$ & $\begin{array}{c}8.2815(2) \\
3.64225(10) \\
-\end{array}$ \\
\hline 2 & $\begin{array}{l}\mathrm{Hf}_{33} \mathrm{Re}_{10} \mathrm{Si}_{57} \\
\mathrm{Hf}_{21.9} \mathrm{Re}_{9.8} \mathrm{Si}_{68.3}\end{array}$ & $\begin{array}{l}\mathbf{H f S i}_{2} \\
\mathrm{Hf}_{17.4(4)} \mathrm{Re}_{12.5(2)} \mathrm{Si}_{70.1(4)} \\
\mathbf{R e}_{4} \mathbf{S i}_{7} \\
\mathrm{Hf}_{23.8(7)} \mathrm{Re}_{16.8(4)} \mathrm{Si}_{59.4(5)} \\
\mathbf{T 1} \\
\mathrm{Hf}_{23(2)} \mathrm{Re}_{4(1)} \mathrm{Si}_{73(1)}\end{array}$ & $\begin{array}{l}o S 12 \\
m S 44\end{array}$ & $\begin{array}{c}\mathrm{Cmcm} \\
\mathrm{Cm}\end{array}$ & $\begin{array}{l}3.6727(2) \\
23.110(4)\end{array}$ & $\begin{array}{c}14.5321(9) \\
3.1383(4) \\
\beta=92.79(2)^{\circ}\end{array}$ & $\begin{array}{l}3.6415(2) \\
8.294(1)\end{array}$ \\
\hline 3 & $\begin{array}{l}\mathrm{Hf}_{57} \mathrm{Re}_{10} \mathrm{Si}_{33} \\
\mathrm{Hf}_{51.1} \mathrm{Re}_{10.4} \mathrm{Si}_{38.5}\end{array}$ & $\begin{array}{l}\mathbf{H f}_{\mathbf{5}} \mathrm{Si}_{\mathbf{3}} \\
\mathrm{Hf}_{52.7(6)} \mathrm{Re}_{1.4(1)} \mathrm{Si}_{45.9(7)} \\
\mathbf{H f R e}_{2} \\
\mathrm{Hf}_{44.9(4)} \operatorname{Re}_{19.4(7)} \mathrm{Si}_{35.7(3)}\end{array}$ & $\begin{array}{l}h P 16 \\
h P 12\end{array}$ & $\begin{array}{l}\mathrm{PG}_{3} / \mathrm{mcm} \\
\mathrm{PG}_{3} / \mathrm{mmc}\end{array}$ & $\begin{array}{l}7.819(1) \\
5.222(2)\end{array}$ & $\begin{array}{l}- \\
-\end{array}$ & $\begin{array}{l}5.476(1) \\
8.513(6)\end{array}$ \\
\hline 4 & $\mathrm{Hf}_{60} \mathrm{Re}_{20} \mathrm{Si}_{20}$ & $\begin{array}{l}\mathbf{H f}_{21} \mathbf{R e}_{25} \\
\mathbf{H f}_{2} \mathbf{S i} \\
\text { unidentified phase }\end{array}$ & $\begin{array}{c}h R 276 \\
t I 12\end{array}$ & $\begin{array}{c}R-3 c \\
I 4 / \mathrm{mcm}\end{array}$ & $\begin{array}{l}25.69(2) \\
6.537(3)\end{array}$ & $\begin{array}{l}- \\
-\end{array}$ & $\begin{array}{l}8.757(8) \\
5.219(4)\end{array}$ \\
\hline 5 & $\mathrm{Hf}_{50} \mathrm{Re}_{30} \mathrm{Si}_{20}$ & $\begin{array}{l}\mathbf{H f R e}_{2} \\
\mathbf{H f}_{2} \mathbf{S i} \\
\text { unidentified phase }\end{array}$ & $\begin{array}{c}h P 12 \\
t I 12\end{array}$ & $\begin{array}{c}\mathrm{PG}_{3} / \mathrm{mmc} \\
\mathrm{I} 4 / \mathrm{mcm}\end{array}$ & $\begin{array}{l}5.226(1) \\
6.335(2)\end{array}$ & - & $\begin{array}{l}8.549(3) \\
5.202(3)\end{array}$ \\
\hline 6 & $\begin{array}{l}\mathrm{Hf}_{33} \mathrm{Re}_{47} \mathrm{Si}_{20} \\
\mathrm{Hf}_{32.9} \mathrm{Re}_{41.1} \mathrm{Si}_{26.0}\end{array}$ & $\begin{array}{l}\text { HfRe }_{2} \\
\mathrm{Hf}_{31.2(6)} \operatorname{Re}_{51.1(6)} \mathrm{Si}_{17.7(1)} \\
\text { HfReSi } \\
\mathrm{Hf}_{31.7(9)} \operatorname{Re}_{28.8(7)} \mathrm{Si}_{39.5(6)} \\
\text { HfSi } \\
\mathrm{Hf}_{43.8(2)} \operatorname{Re}_{11.2(5)} \mathrm{Si}_{45.0(4)}\end{array}$ & $\begin{array}{l}h P 12 \\
h P 9 \\
o P 8\end{array}$ & $\begin{array}{c}P 6_{3} / m m c \\
P-62 m \\
\text { Pnma }\end{array}$ & $\begin{array}{l}5.1899(1) \\
6.9205(8) \\
9.902(4)\end{array}$ & $\begin{array}{c}- \\
- \\
3.776(2)\end{array}$ & $\begin{array}{l}8.5009(3) \\
3.4133(7) \\
5.272(3)\end{array}$ \\
\hline 7 & $\mathrm{Hf}_{33} \mathrm{Re}_{57} \mathrm{Si}_{10}$ & $\mathrm{HfRe}_{2}$ & $h P 12$ & $P 6_{3} / m m c$ & $5.2238(6)$ & - & $8.548(2)$ \\
\hline 8 & $\begin{array}{l}\mathrm{Hf}_{20} \mathrm{Re}_{60} \mathrm{Si}_{20} \\
\mathrm{Hf}_{19.5} \mathrm{Re}_{57.9} \mathrm{Si}_{22.7}\end{array}$ & $\begin{array}{l}\mathbf{H f}_{\mathbf{5}} \mathbf{R e}_{24} \\
\mathrm{Hf}_{14.9(7)} \operatorname{Re}_{73.5(4)} \mathrm{Si}_{11.6(4)} \\
\mathbf{H f R e}_{2} \\
\mathrm{Hf}_{3.3(6)} \operatorname{Re}_{36.6(5)} \mathrm{Si}_{60.1(1)} ? \\
\mathbf{H f R e S i} \\
\mathrm{Hf}_{25.0(4)} \operatorname{Re}_{45(2)} \mathrm{Si}_{30(2)} \\
\end{array}$ & $\begin{array}{l}c I 58 \\
h P 12 \\
h P 9\end{array}$ & $\begin{array}{c}I-43 m \\
P 6_{3} / m m c \\
P-62 m\end{array}$ & $\begin{array}{l}9.597(1) \\
5.145(1) \\
6.92(1)\end{array}$ & $\begin{array}{l}- \\
- \\
-\end{array}$ & $\begin{array}{c}- \\
8.418(3) \\
3.375(6)\end{array}$ \\
\hline 9 & $\mathrm{Hf}_{10} \mathrm{Re}_{57} \mathrm{Si}_{33}$ & $\begin{array}{l}\mathrm{Hf}_{5} \mathrm{Re}_{24} \\
\text { ReSi } \\
\text { HfReSi }\end{array}$ & $\begin{array}{l}c I 58 \\
c P 8 \\
h P 9 \\
\end{array}$ & $\begin{array}{c}I-43 m \\
P 2_{1} 3 \\
P-62 m\end{array}$ & $\begin{array}{c}9.5135(8) \\
4.7765(4) \\
6.903(3) \\
\end{array}$ & $\begin{array}{l}- \\
- \\
-\end{array}$ & $\begin{array}{c}- \\
- \\
3.352(2) \\
\end{array}$ \\
\hline 10 & $\begin{array}{l}\mathrm{Hf}_{25} \mathrm{Re}_{25} \mathrm{Si}_{50} \\
\mathrm{Hf}_{24.3} \mathrm{Re}_{24.7} \mathrm{Si}_{51.0}\end{array}$ & $\begin{array}{l}\text { HfReSi } \\
\mathrm{Hf}_{29.1(3)} \operatorname{Re}_{30.3(5)} \mathrm{Si}_{40.6(8)} \\
\mathbf{H f R e S i}_{2} \\
\mathrm{Hf}_{21.6(4)} \operatorname{Re}_{21.8(4)} \mathrm{Si}_{56.6(1)} \\
\mathbf{R e}_{4} \mathbf{S i}_{7} \\
\mathrm{Hf}_{7(1)} \operatorname{Re}_{30.7(9)} \mathrm{Si}_{62.3(4)} \\
\end{array}$ & $\begin{array}{c}h P 9 \\
o P 48 \\
m S 44\end{array}$ & $\begin{array}{c}\text { P-62m } \\
\text { Pbam } \\
\text { Cm }\end{array}$ & $\begin{array}{l}6.921(2) \\
9.119(3) \\
23.2(4)\end{array}$ & $\begin{array}{c}- \\
10.027(3) \\
3.126(3) \\
\beta=92.7(1)^{\circ}\end{array}$ & $\begin{array}{l}3.385(2) \\
8.064(3) \\
8.28(1)\end{array}$ \\
\hline 11 & $\mathrm{Hf}_{33.3} \mathrm{Re}_{33.3} \mathrm{Si}_{33.4}$ & $\begin{array}{l}\text { HfReSi } \\
\text { HfRe }_{2} \\
\text { unidentified phase } \\
\end{array}$ & $\begin{array}{c}h P 9 \\
h P 12\end{array}$ & $\begin{array}{c}P-62 m \\
P 6_{3} / m m c\end{array}$ & $\begin{array}{l}6.9218(2) \\
5.257(2)\end{array}$ & $\begin{array}{l}- \\
- \\
- \\
\end{array}$ & $\begin{array}{l}3.3898(1) \\
8.592(5)\end{array}$ \\
\hline 12 & $\begin{array}{l}\mathrm{Hf}_{64} \mathrm{Re}_{29} \mathrm{Si}_{7} \\
\mathrm{Hf}_{62.2} \mathrm{Re}_{31.6} \mathrm{Si}_{6.2}\end{array}$ & $\begin{array}{l}\mathbf{H f}_{\mathbf{2 1}} \mathbf{R e}_{25} \\
\mathrm{Hf}_{43(3)} \operatorname{Re}_{51(2)} \mathrm{Si}_{6(1)} \\
\mathbf{H f}_{2} \mathrm{Si} \\
\mathrm{Hf}_{64(3)} \operatorname{Re}_{3(1)} \mathrm{Si}_{33(2)} \\
\mathbf{T 2} \\
\mathrm{Hf}_{71(2)} \mathrm{Re}_{18(3)} \mathrm{Si}_{11(1)}\end{array}$ & $\begin{array}{c}h R 276 \\
t I 12\end{array}$ & $\begin{array}{c}R-3 c \\
I 4 / m c m\end{array}$ & $\begin{array}{c}25.676(9) \\
6.516(6)\end{array}$ & $\begin{array}{l}- \\
-\end{array}$ & $\begin{array}{l}8.757(5) \\
5.261(9)\end{array}$ \\
\hline
\end{tabular}




\section{Conclusions}

The system $\mathrm{Hf}-\mathrm{Re}-\mathrm{Si}$ at $1000^{\circ} \mathrm{C}$ is characterized by significant solubility of the third component in the binary phases. In addition to the already known compounds, two new ternary phases with approximate compositions $\quad \mathrm{Hf}_{23(2)} \mathrm{Re}_{4(1)} \mathrm{Si}_{73(1)} \quad$ (T1) and $\mathrm{Hf}_{71(2)} \mathrm{Re}_{18(3)} \mathrm{Si}_{11(1)}$ (T2) were observed. Their structures will be the topic of a future publication.

\section{Acknowledgement}

This work was supported by the Ministry of Education and Science of Ukraine under the grant No. 0118 U003609.

\section{References}

[1] I. Engström, B. Lönnberg, J. Appl. Phys. 63 (1988) 4476-4484.

[2] SpringerMaterials (https://materials.springer.com).

[3] P. Villars, K. Cenzual (Eds.), Pearson's Crystal Data - Crystal Structure Database for Inorganic Compounds, Release 2017/18, ASM International, Materials Park, OH, 2017.

[4] Y.P. Yarmolyuk, E.I. Gladyshevskii, Dopov. Akad. Nauk Ukr. RSR, Ser. B (1974) 1030-1032.

[5] Y.P. Yarmolyuk, M. Sikiritsa, L.G. Akselrud, L.A. Lysenko, E.I. Gladyshevskii, Sov. Phys. Crystallogr. 27 (1982) 652-653.

[6] P. Villars, H. Okamoto, K. Cenzual (Eds.), ASM Alloy Phase Diagram Database, Release 2006/2018, ASM International, Materials Park, $\mathrm{OH}, 2018$.

[7] L. Akselrud, Yu. Grin, J. Appl. Crystallogr. 47 (2014) 803-805.

[8] K. Cenzual, E. Parthé, R.M. Waterstrat, Acta Crystallogr. C 42 (1986) 261-266.

[9] V.B. Compton, B.T. Matthias, Acta Crystallogr. 12 (1959) 651-654.

[10] E.M. Savitskii, M.A. Tylkina, I.A. Tsyganova, E.I. Gladyshevskii, M.P. Mulyava, Russ. J. Inorg. Chem. 7 (1962) 831-832.
[11] E.E. Havinga, H. Damsma, P. Hokkeling, J. Less-Common Met. 27 (1972) 169-186.

[12] O.G. Karpinskii, B.A. Evseyev, Russ. Metall. (1969) 128-130.

[13] T. Siegrist, J.E. Greedan, J.D. Garrett, G. Wenhe, C.V. Stager, J. Less-Common Met. 171 (1991) 171-177.

[14] A.G. Knapton, Plansee Proc. Pap. Plansee Semin. “de Re Met.”, $3^{\text {rd }}$, 1959, pp. 412-418.

[15] V.M. Fedyna, R.E. Gladyshevskii, Visn. Lviv. Univ., Ser. Khim. 55 (2014) 87-92.

[16] S. Harada, H. Hoshikawa, K. Kuwabara, K. Tanaka, E. Okunishi, H. Inui, Philos. Mag. 91 (2011) 3108-3127.

[17] U. Gottlieb, B. Lambert Andron, F. Nava, M. Affronte, O. Laborde, A. Rouault, R. Madar, J. Appl. Phys. 78 (1995) 3902-3907.

[18] J.L. Jorda, M. Ishikawa, J. Muller, J. LessCommon Met. 85 (1982) 27-35.

[19] T. Siegrist, F. Hulliger, G. Travaglini, J. LessCommon Met. 92 (1983) 119-129.

[20] T.B. Massalski (Ed.), Binary Alloy Phase Diagrams, Second Edition, ASM International, Materials Park, Ohio, 1990, Vol. 3, pp. 21052106.

[21] T.B. Massalski (Ed.), Binary Alloy Phase Diagrams, Second Edition, ASM International, Materials Park, Ohio, 1990, Vol. 3, pp. 21112112.

[22] T.B. Massalski (Ed.), Binary Alloy Phase Diagrams, Second Edition, ASM International, Materials Park, Ohio, 1990, Vol. 3, pp. 32043205.

[23] E. Brukl, Tech. Rep. AFML-TR-65-2, Air Force Materials Laboratory, WPAFB (OH), 1968, 72 p.

[24] A.K. Shurin, N. Todorov, Metallofiz. 33 (1971) 100-102.

[25] R. Kieffer, F. Benesovsky, Powder Metall. (1/2) (1958) 145-171.

[26] H. Nowotny, E. Laube, T. Kieffer, F. Benesovsky, Monatsh. Chem. 89 (1958) 701-707.

[27] B.P. Bewlay, J.A. Sutliff, R.R. Bishop, J. Phase Equilib. 20 (1999) 109-112. 